\title{
Zolimomab Aritox
}

National Cancer Institute

\section{Source}

National Cancer Institute. Zolimomab Aritox. NCI Thesaurus. Code C29549.

An immunotoxin consisting of a murine anti-CD5 monoclonal antibody conjug ated to the ricin A chain. The anti-CD5 monoclonal antibody component binds to CD5, a T-cell associated antigen, thereby targeting the delivery of the cytotoxic ricin A chain to T-cells. The ricin A chain component binds to T-cells and disrupts ribosomal activity and protein synthesis and induces cell cycle arrest. ( $\mathrm{NClO4})$ 